\title{
Adomian Decomposition Method for Solving Goursat's Problems
}

\author{
Mariam A. Al-Mazmumy \\ Mathematics Department, Science College, King Abdulaziz University, Jeddah, Saudi Arabia \\ E-mail:mome0505@hotmail.com \\ Received May 18, 2011; revised June 22, 2011; accepted June 29, 2011
}

\begin{abstract}
In this paper, Goursat's problems for: linear and nonlinear hyperbolic equations of second-order, systems of nonlinear hyperbolic equations and fourth-order linear hyperbolic equations in which the attached conditions are given on the characteristics curves are transformed in such a manner that the Adomian decomposition method (ADM) can be applied. Some examples with closed-form solutions are studied in detail to further illustrate the proposed technique, and the results obtained indicate this approach is indeed practical and efficient.
\end{abstract}

Keywords: Goursat’s Problem, Linear and Nonlinear Hyperbolic Equation of Second and Fourth-Orders, System of Linear Hyperbolic Equations of Second Order, Adomian Decomposition Method

\section{Introduction}

The simple Goursat's problem concerns a class of linear hyperbolic equations of second-order in two independent variables with given values on two characteristics curves [1]. For example, for the linear hyperbolic equation

$$
u_{t t}-u_{x x}+\lambda u=f(t, x)
$$

Goursat's problem is posed as follows:

$$
\begin{cases}u_{t t}-u_{x x}+\lambda u=f(t, x), & \lambda \in \mathfrak{R} \\ u(t, x)=\varphi_{0}(x) & \text { for } x-t=0 \\ u(t, x)=\varphi_{1}(x) & \text { for } x+t=0\end{cases}
$$

Several numerical methods such as Range-Kutta method, finite difference method and finite elements method have been used to approach the problem.

The general difficult which arises to us is the presence of the attached conditions on two characteristics curves $x-t=0$ and $x+t=0$ which complicates the application of numerical methods and Adomian decomposition method [2-12].

The clue of this one consists in transforming this type of problems into classical problems where the conditions can be converted into initial conditions. In this technique, we use the variables $w=x+t$ and $z=x-t$. Hence we show that the linear Goursat models will be approached more effectively and rapidly by using the Adomian decomposition method (ADM) to obtain the exact solutions to this type of problems. In a manner parallel to this problem, we study a class of Goursat's problems for nonlinear hyperbolic equations, systems of nonlinear hyperbolic equations and fourth-order linear hyperbolic equations. Our techniques are easily applicable and offer a very direct way to determine the solutions.

The present paper extends some results of [13].

\section{The Goursat's Problem for Linear Hyperbolic Equations of Second Order}

In this section consider Goursat's problem (1).

Our first approach consists in converting problem (1) into a classical problem by introducing new variables $w=x+t$ and $z=x-t$. The second one is that in order to find the solution for the given problem we consider this form as the most general form for ADM.

Make the substitutions $w=x+t$ and $z=x-t$. into the first Equation of (1), and applying the chain rule to obtain

$$
\begin{gathered}
\frac{\partial u}{\partial x}=\frac{\partial u}{\partial w}+\frac{\partial u}{\partial z} \\
\frac{\partial u}{\partial t}=\frac{\partial u}{\partial w}-\frac{\partial u}{\partial z} \\
\frac{\partial^{2} u}{\partial x^{2}}=\frac{\partial^{2} u}{\partial w^{2}}+2 \frac{\partial^{2} u}{\partial w \partial z}+\frac{\partial^{2} u}{\partial z^{2}}
\end{gathered}
$$




$$
\frac{\partial^{2} u}{\partial t^{2}}=\frac{\partial^{2} u}{\partial w^{2}}-2 \frac{\partial^{2} u}{\partial w \partial z}+\frac{\partial^{2} u}{\partial z^{2}}
$$

Then the first Equation of (1) becomes

$$
-4 \frac{\partial^{2} u}{\partial w \partial z}=f\left(\frac{w-z}{2}, \frac{w+z}{2}\right)-\lambda u
$$

Also the given conditions of (1) can be converted into

$$
\begin{aligned}
& u(w, 0)=\varphi_{0}\left(\frac{w}{2}\right) \\
& u(0, z)=\varphi_{1}\left(\frac{z}{2}\right)
\end{aligned}
$$

Then,

Lemma 1. Goursat's problem (1) is equivalent to (2)-(4).

Now we shall use the Adomian's decomposition method [2-12] for solving (2)-(4).

Consider Equation (2) in an operator form as

$$
L_{w z} u=-\frac{1}{4} f+\frac{\lambda}{4} u
$$

where $L_{w z}(u)=\frac{\partial^{2} u}{\partial w \partial z}$

Operating with the inverse operator $L_{w z}^{-1}()=.\int_{0}^{w} \int_{0}^{z}(.) \mathrm{d} w \mathrm{~d} z$, we have

$$
\begin{aligned}
u(w, z) & =\varphi_{0}\left(\frac{w}{2}\right)+\phi_{1}\left(\frac{z}{2}\right)-u(0,0) \\
& -\frac{1}{4} \int_{0}^{w} \int_{0}^{z} f\left(\frac{w-z}{2}, \frac{w+z}{2}\right) \mathrm{d} w \mathrm{~d} z \\
& +\frac{\lambda}{4} \int_{0}^{w} \int_{0}^{z} u(w, z) \mathrm{d} w \mathrm{~d} z
\end{aligned}
$$

where $u(0,0)=\varphi_{0}(0)=\varphi_{1}(0)$.

Following the Adomian decomposition method the unknown solution $u$ is assumed to be given by a series of the form $u(w, z)=\sum_{n=0}^{\infty} u_{n}(w, z)$ where the components $u_{n}(w, z)$ are going to be determined recurrently.

Thus, we get the scheme

$$
\left\{\begin{aligned}
u_{0} & =\varphi_{0}\left(\frac{w}{2}\right)+\varphi_{1}\left(\frac{z}{2}\right)-u(0,0) \\
& -\frac{1}{4} \int_{0}^{w} \int_{0}^{z} f\left(\frac{w-z}{2}, \frac{w+z}{2}\right) \mathrm{d} w \mathrm{~d} z \\
u_{n} & =\frac{\lambda}{4} \int_{0}^{w} \int_{0}^{z} u_{n-1} \mathrm{~d} w \mathrm{~d} z, n \geq 1
\end{aligned}\right.
$$

Now, by summing the first $n+1$ terms of $u(w, z)=\sum_{n=0}^{\infty} u_{n}(w, z)$ we obtain the $n t h$ approximation to the solution $\varphi$ as

$$
S_{n}=\sum_{i=0}^{n} u_{i}, n \geq 0
$$

or

$$
S_{n}=u_{0}+\sum_{i=1}^{n} u_{i}
$$

By substitution of the recursive scheme (7) into this sum, we conclude that the ADM for Goursat's problem (1) can be converted to an equivalent problem, which we state as follows

Theorem 1. The ADM for Goursat's problem (1) is equivalent to the following problem:

Find the sequence $S_{n}$ such that $S_{n}=u_{0}+u_{1}+\cdots+u_{n}$ and satisfies

$$
\left\{\begin{aligned}
u_{0}= & \varphi_{0}\left(\frac{w}{2}\right)+\varphi_{1}\left(\frac{z}{2}\right)-u(0,0) \\
& -\frac{1}{4} \int_{0}^{w} \int_{0}^{z} f\left(\frac{w-z}{2}, \frac{w+z}{2}\right) \mathrm{d} w \mathrm{~d} z \\
S_{n}= & u_{0}+\frac{\lambda}{4} \int_{0}^{w} \int_{0}^{z} S_{n-1} \mathrm{~d} w \mathrm{~d} z, n \geq 1
\end{aligned}\right.
$$

Also, the result concerning the convergence analysis of the ADM for problem (1) can be stated as follows

Theorem 2. Let $S_{n}$ be a sequence defined by (10). If $\left\|u_{0}\right\|<\infty$ and there exist a constant $0 \leq \alpha<1$, such that $\left\|u_{n+1}\right\| \leq \alpha\left\|u_{n}\right\|$ then $S_{n}$ is convergent.

Proof. We have

$$
\left\|S_{n}-S_{m}\right\|=\left\|S_{n}-S_{n-1}+S_{n-1}-S_{n-2}+S_{n-2}+S_{m+1}-S_{m}\right\|
$$

for $n \geq m$, then

$$
\left\|S_{n}-S_{m}\right\| \leq\left\|S_{n}-S_{n-1}\right\|+\left\|S_{n-1}-S_{n-2}\right\|+\ldots+\left\|S_{m+1}-S_{m}\right\|
$$

Thus

$$
\left\|S_{n}-S_{m}\right\| \leq \alpha^{n}\left\|u_{0}\right\|+\alpha^{n-1}\left\|u_{0}\right\|+\cdots+\alpha^{m+1}\left\|u_{0}\right\|
$$

Therefore

$$
\left\|S_{n}-S_{m}\right\| \leq \alpha^{m+1}\left(1+\alpha+\cdots+\alpha^{n-m-1}\right)\left\|u_{0}\right\|
$$

As $\sum_{i=0}^{\infty} \alpha^{i}$ is a geometric series with the common ratio $\alpha, \quad 0 \leq \alpha<1$. By induction we see that

$$
\left\|S_{n}-S_{m}\right\| \leq \frac{\alpha^{m+1}}{1-\alpha}\left\|u_{0}\right\|
$$

and $\left\|S_{n}-S_{m}\right\| \rightarrow 0$, as $m \rightarrow \infty$, thus $S_{n}$ converges for $0 \leq \alpha<1$ so we have convergence in norm. 


\section{The Goursat's Problem for Nonlinear Hyperbolic Equations of the Second Order}

The same procedure can be adopted to resolve the following nonlinear hyperbolic equation of the second order

$$
\begin{cases}u_{t t}-u_{x x}+\lambda u=f(t, x)+F(u) \\ u(t, x)=\varphi_{0}(x) & \text { for } x-t=0 \\ u(t, x)=\varphi_{1}(x) & \text { for } x+t=0\end{cases}
$$

where the nonlinear term is represented by $F(u)$. As before, we have

Lemma 2. The nonlinear Goursat's problem (11) is equivalent to

$$
\left\{\begin{array}{l}
-4 \frac{\partial^{2} u}{\partial w \partial z}=f\left(\frac{w-z}{2}, \frac{w+z}{2}\right)-\lambda u+F(u) \\
u(w, 0)=\varphi_{0}\left(\frac{w}{2}\right) \\
u(0, z)=\varphi_{1}\left(\frac{z}{2}\right)
\end{array}\right.
$$

Substituting the decomposition $u(w, z)=\sum_{n=0}^{\infty} u_{n}(w, z)$ into (12) and define $F(u)$ by $F(u)=\sum_{n=0}^{\infty} A_{n}\left(u_{0}, u_{1}, \cdots, u_{n}\right)$ where the $A_{n}$ are the Adomian polynomials [2,3], which depend only on the components $u_{0}, u_{1}, \cdots, u_{n}$. Thus we get the recursive scheme

$$
\left\{\begin{array}{l}
u_{0}=\varphi_{0}\left(\frac{w}{2}\right)+\varphi_{1}\left(\frac{z}{2}\right)-u(0,0) \\
u_{n}=\frac{\lambda}{4} \int_{0}^{w} \int_{0}^{z} u_{n-1} \mathrm{~d} w \mathrm{~d} z--\frac{1}{4} \int_{0}^{w} \int_{0}^{z} A_{n-1} \mathrm{~d} w \mathrm{~d} z, n \geq 1
\end{array}\right.
$$

As before, we conclude that the ADM for Goursat's problem (11) can be converted to an equivalent problem.

Theorem 3. The ADM for Goursat's problem (11) is equivalent to the following problem:

Find the sequence $S_{n}$ such that $S_{n}=u_{0}+u_{1}+\cdots+u_{n}$ and satisfies

$$
\left\{\begin{array}{c}
u_{0}=\varphi_{0}\left(\frac{w}{2}\right)+\varphi_{1}\left(\frac{z}{2}\right)-u(0,0) \\
-\frac{1}{4} \int_{0}^{w} \int_{0}^{z} f\left(\frac{w-z}{2}, \frac{w+z}{2}\right) \mathrm{d} w \mathrm{~d} z \\
S_{n}=u_{0}+\frac{\lambda}{4} \int_{0}^{w} \int_{0}^{z} F\left(S_{n-1}\right) \mathrm{d} w \mathrm{~d} z, n \geq 1
\end{array}\right.
$$

\section{The Goursat's Problem for System of Nonlinear Hyperbolic Equations of the Second Order}

We shall extend this method to the following system of nonlinear hyperbolic equations of the second order

$$
\left\{\begin{array}{l}
u_{t t}-u_{x x}+F_{1}(u, v)=f_{1}(t, x) \\
v_{t t}-v_{x x}+F_{2}(u, v)=f_{2}(t, x) \\
u(t, x)=\varphi_{0}(x) \text { for } x-t=0 \\
u(t, x)=\varphi_{1}(x) \text { for } x+t=0 \\
v(t, x)=\psi_{0}(x) \text { for } x-t=0 \\
v(t, x)=\psi_{1}(x) \text { for } x+t=0
\end{array}\right.
$$

where the nonlinear terms are represented by $F_{i}(u, v)$, $i=1,2$.

By the same way as given in the nonlinear hyperbolic Equation (11), the scheme for system (15) can be expressed as

$$
\left\{\begin{aligned}
u_{0} & =\varphi_{0}\left(\frac{w}{2}\right)+\varphi_{1}\left(\frac{z}{2}\right)-u(0,0) \\
& -\frac{1}{4} \int_{0}^{w} \int_{0}^{z} f_{1}\left(\frac{w-z}{2}, \frac{w+z}{2}\right) \mathrm{d} w \mathrm{~d} z \\
v_{0} & =\psi_{0}\left(\frac{w}{2}\right)+\psi_{1}\left(\frac{z}{2}\right)-v(0,0) \\
& -\frac{1}{4} \int_{0}^{w} \int_{0}^{z} f_{2}\left(\frac{w-z}{2}, \frac{w+z}{2}\right) \mathrm{d} w \mathrm{~d} z \\
u_{n} & =\frac{1}{4} \int_{0}^{w} \int_{0}^{z} A_{n-1} \mathrm{~d} w \mathrm{~d} z, n \geq 1 \\
v_{n} & =\frac{1}{4} \int_{0}^{w} \int_{0}^{z} B_{n-1} \mathrm{~d} w \mathrm{~d} z, n \geq 1
\end{aligned}\right.
$$

where $F_{1}(u, v)=\sum_{n=0}^{\infty} A_{n}\left(u_{0}, u_{1}, \cdots, u_{n} ; v_{0}, v_{1}, \cdots, v_{n}\right)$ and $F_{2}(u, v)=\sum_{n=0}^{\infty} B_{n}\left(u_{0}, u_{1}, \cdots, u_{n} ; v_{0}, v_{1}, \cdots, v_{n}\right)$ and $A_{n}$ and $B_{n}$ are the Adomian polynomials [2,3].

\section{The Goursat's Problem for Linear Hyperbolic Equation of Fourth Order}

In a manner parallel to the previous section, we study a linear hyperbolic equation of fourth-order with multiple characteristics curves. The Goursat's problem for this equation is formulated as follows: 


$$
\left(\frac{\partial^{2}}{\partial t^{2}}-\frac{\partial^{2}}{\partial x^{2}}\right)^{2} u+\lambda u=f(t, x)
$$

To Equation (17), we attach the following conditions

$$
\begin{array}{ll}
u(t, x)=\varphi_{0}(x) & \text { for } x-t=0 \\
\frac{\partial u}{\partial t}-\frac{\partial u}{\partial x}=\varphi_{1}(x) & \text { for } x-t=0 \\
u(t, x)=\varphi_{2}(x) & \text { for } x+t=0
\end{array}
$$

and

$$
\frac{\partial u}{\partial t}+\frac{\partial u}{\partial x}=\varphi_{3}(x) \quad \text { for } x+t=0
$$

We mention that the attached conditions are given on the multiple characteristics curves $x-t=0$ and $x+t=0$ of Equation (17)

Let $u$ be a solution of problem (18)-(21), make the transformations $z=x-t, w=x+t$, and applying the chain rule to obtain

$$
\frac{\partial^{4} u}{\partial w^{2} \partial z^{2}}=\frac{1}{16} f\left(\frac{w-z}{2}, \frac{w+z}{2}\right)-\frac{\lambda}{16} u
$$

and the given conditions (18)-(21) can be converted into

$$
\begin{aligned}
& u(w, 0)=\varphi_{0}\left(\frac{w}{2}\right) \\
& \frac{\partial u}{\partial z}(w, 0)=-\frac{1}{2} \varphi_{0}\left(\frac{w}{2}\right) \\
& u(0, z)=\varphi_{2}\left(\frac{z}{2}\right) \\
& \frac{\partial u}{\partial w}(0, z)=\frac{1}{2} \varphi_{3}\left(\frac{z}{2}\right)
\end{aligned}
$$

Then,

Lemma 3. Goursat's problem (17)-(21) is now equivalent to (22)-(26).

As before, rewrite Equation (22) in an operator form as

$$
L_{w w z z} u=\frac{1}{16} f-\frac{\lambda}{16} u
$$

where $L_{w w z z}(u)=\frac{\partial^{4} u}{\partial w^{2} \partial z^{2}}$

Operating with the inverse operator

$$
L_{w w z z}^{-1}(.)=\int_{0}^{w} \int_{0}^{w} \int_{0}^{z} \int_{0}^{z}(.) \mathrm{d} w \mathrm{~d} w \mathrm{~d} z \mathrm{~d} z
$$

$$
\begin{aligned}
u(w, z) & =u(w, 0)+u(0, z)-u(0,0) \\
& +z \frac{\partial u}{\partial z}(w, 0)+w \frac{\partial u}{\partial w}(0, z)-z \frac{\partial u}{\partial z}(0,0) \\
& -w \frac{\partial u}{\partial w}(0,0)-w z \frac{\partial^{2} u}{\partial w \partial z}(0,0) \\
& +\frac{1}{16} \int_{0}^{w} \int_{0}^{z} \int_{0}^{w} \int_{0}^{z} f\left(\frac{w-z}{2}, \frac{w+z}{2}\right) \mathrm{d} w \mathrm{~d} w \mathrm{~d} z \mathrm{~d} z \\
& -\frac{\lambda}{16} \int_{0}^{w} \int_{0}^{z} \int_{0}^{w} \int_{0}^{z} u(w, z) \mathrm{d} w \mathrm{~d} w \mathrm{~d} z \mathrm{~d} z
\end{aligned}
$$

where

$$
\begin{gathered}
u(0,0)=\varphi_{0}(0)=\varphi_{2}(0) \\
\frac{\partial^{2} u}{\partial w \partial z}(0,0)=-\frac{1}{4} \varphi_{1}(0) \\
\frac{\partial u}{\partial z}(0,0)=-\frac{1}{2} \varphi_{1}(0)
\end{gathered}
$$

and

$$
\frac{\partial u}{\partial w}(0,0)=\frac{1}{2} \varphi_{3}(0)
$$

So that

$$
\begin{aligned}
u(x, t) & =\varphi_{0}\left(\frac{w}{2}\right)+\varphi_{2}\left(\frac{z}{2}\right)-u(0,0) \\
& -\frac{z}{2} \varphi_{1}\left(\frac{w}{2}\right)+\frac{w}{2} \varphi_{3}\left(\frac{z}{2}\right)+\frac{z}{2} \varphi_{1}(0) \\
& -\frac{w}{2} \varphi_{3}(0)+\frac{w z}{4} \varphi_{1}^{\prime}(0) \\
& +\frac{1}{16} \int_{0}^{w} \int_{0}^{z} \int_{0}^{w} \int_{0}^{z} f\left(\frac{w-z}{2}, \frac{w+z}{2}\right) \mathrm{d} w \mathrm{~d} w \mathrm{~d} z \mathrm{~d} z \\
& -\frac{\lambda}{16} \int_{0}^{w} \int_{0}^{z} \int_{0}^{w} \int_{0}^{z} u(w, z) \mathrm{d} w \mathrm{~d} w \mathrm{~d} z \mathrm{~d} z
\end{aligned}
$$

Following the Adomian decomposition method we get the scheme

$$
\left\{\begin{array}{l}
u_{0}=\psi(w, z) \\
u_{n}=-\frac{\lambda}{16} \int_{0}^{w} \int_{0}^{z} \int_{0}^{w} \int_{0}^{z} u_{n-1} \mathrm{~d} w \mathrm{~d} w \mathrm{~d} z \mathrm{~d} z, n \geq 1
\end{array}\right.
$$

where

$$
\begin{aligned}
\psi(w, z) & =\varphi_{0}\left(\frac{w}{2}\right)+\varphi_{2}\left(\frac{z}{2}\right)-u(0,0) \\
& -\frac{z}{2} \varphi_{1}\left(\frac{w}{2}\right)+\frac{w}{2} \varphi_{3}\left(\frac{z}{2}\right)+\frac{z}{2} \varphi_{1}(0) \\
& -\frac{w}{2} \varphi_{3}(0)+\frac{w z}{4} \varphi_{1}^{\prime}(0)+\mu(w, z)
\end{aligned}
$$


and

$$
\mu(w, z)=\frac{1}{16} \int_{0}^{w} \int_{0}^{z} \int_{0}^{w} \int_{0}^{z} f(t, x) \mathrm{d} w \mathrm{~d} w \mathrm{~d} z \mathrm{~d} z
$$

\section{Applications}

In order to illustrate a possible practical use of the ADM, we shall give in this section some examples.

Example 1. Consider problem (1) with

$$
\begin{aligned}
& u(t, x)=\varphi_{0}(0)=0 \quad \text { for } x-t=0 \\
& u(t, x)=\varphi_{1}(0)=0 \quad \text { for } x+t=0 \\
& f(t, x)=-4 \text { and } \lambda=0
\end{aligned}
$$

Then by the above recurrent scheme, we obtain

$$
\left\{\begin{array}{l}
u_{0}=\int_{0}^{w} \int_{0}^{z} \mathrm{~d} w \mathrm{~d} z=w z \\
u_{n}=0, n \geq 1
\end{array}\right.
$$

So $u=\sum_{n=0}^{\infty} u_{n}(w, z)$ can be determined as $u(w, z)=w z$. We return to the original variables $w=x+t$ and $z=x-t$ we get $u(t, x)=x^{2}-t^{2}$ which is the exact solution.

Example 2. In problem (1) we choose

$$
\begin{array}{ll}
u(t, x)=\varphi_{0}(0)=2 x & \text { for } x-t=0 \\
u(t, x)=\varphi_{1}(0)=2 x & \text { for } x+t=0 \\
f(t, x)=-2 x \quad \text { and } & \lambda=1
\end{array}
$$

Then by the above recurrent scheme, we obtain

$$
\left\{\begin{array}{l}
u_{0}=w+z+\frac{1}{4}\left(\frac{w^{2}}{2} z+\frac{z^{2}}{2} w\right) \\
u_{1}=-\frac{1}{4}\left(\frac{w^{2}}{2} z+\frac{z^{2}}{2} w\right)-\frac{1}{4^{2}}\left(\frac{w^{3}}{6} \frac{z^{2}}{2}+\frac{z^{3}}{6} \frac{w^{2}}{2}\right) \\
u_{2}=\frac{1}{4^{2}}\left(\frac{w^{3}}{6} \frac{z^{2}}{2}+\frac{z^{3}}{6} \frac{w^{2}}{2}\right)+\frac{1}{4^{3}}\left(\frac{w^{4}}{24} \frac{z^{3}}{6}+\frac{z^{4}}{24} \frac{w^{3}}{6}\right) \\
\cdots \\
u_{n}=\frac{(-1)^{n}}{4^{n}}\left[\frac{w^{n+1}}{(n+1) !} \frac{z^{n}}{n !}+\frac{z^{n+1}}{(n+1) !} \frac{w^{n}}{n !}\right] \\
+\frac{(-1)^{n}}{4^{n+1}}\left[\frac{w^{n+2}}{(n+2) !} \frac{z^{n+1}}{(n+1) !}+\frac{z^{n+2}}{(n+2) !} \frac{w^{n+1}}{(n+1) !}\right], n \geq 1
\end{array}\right.
$$

So $u=\sum_{n=0}^{\infty} u_{n}(w, z)$ can be determined as $u(w, z)=w+z$. Thus $u(t, x)=2 x$ which is the exact solution.

Example 3. Consider now the Goursat's problem (17)-(21) with

$$
\begin{gathered}
u(t, x)=\varphi_{0}(x)=x^{4} \quad \text { for } \quad x-t=0 \\
\frac{\partial u}{\partial t}-\frac{\partial u}{\partial x}=\varphi_{1}(x)=x^{2} \quad \text { for } x-t=0 \\
u(t, x)=\varphi_{2}(x)=\frac{1}{4} x^{2} \text { for } x+t=0 \\
\frac{\partial u}{\partial t}+\frac{\partial u}{\partial x}=\varphi_{3}(x)=0 \quad \text { for } x+t=0 \\
f(t, x)=0 \quad \text { and } \quad \lambda=0
\end{gathered}
$$

The recurrence relation successively yields

$$
\left\{\begin{array}{l}
u_{0}=\left(\frac{w}{2}\right)^{4}+\frac{1}{4}\left(\frac{z}{2}\right)^{2}-\frac{z}{2}\left(\frac{w}{2}\right)^{2}=\frac{1}{16}\left(w^{4}+z^{2}\right)-\frac{1}{8} z w^{2} \\
u_{n}=0, n \geq 1
\end{array}\right.
$$

Then $u(w, z)=\frac{1}{16}\left(w^{4}+z^{2}\right)-\frac{1}{8} z w^{2}$ Hence, direct calculation produces the exact solution

$$
u(t, x)=\frac{1}{16}\left[(x+t)^{2}-(x-t)\right]^{2}
$$

Example 4. Consider Goursat's problem (15) with

$$
\begin{gathered}
\varphi_{0}(x)=\sin 2 x \text { for } x-t=0 \\
\varphi_{1}(x)=0 \text { for } x+t=0 \\
\psi_{0}(x)=\cos 2 x \text { for } x-t=0 \\
\psi_{1}(x)=1 \text { for } x+t=0 \\
f_{i}(t, x)=0 \text { and } F_{i}(u, v)=u^{2}+v^{2}-1, i=1,2
\end{gathered}
$$

Then by the above recurrent scheme, we obtain

$$
\left\{\begin{array}{l}
u_{0}=\sin w \\
v_{0}=\cos w \\
u_{n}=0, n \geq 1 \\
v_{n}=0, n \geq 1
\end{array}\right.
$$

where the first few Adomian's polynomial of the nonlinear terms $F_{i}(u, v)=u^{2}+v^{2}-1, i=1,2$ can be expressed as

$$
\begin{gathered}
A_{0}=B_{0}=u_{0}^{2}+v_{0}^{2}-1 \\
A_{1}=B_{1}=2 u_{0} u_{1}+2 v_{0} v_{1} \\
A_{2}=B_{2}=2 u_{0} u_{2}+u_{1}^{2}+2 v_{0} v_{2}+v_{1}^{2}, \cdots
\end{gathered}
$$


Thus $u=\sum_{n=0}^{\infty} u_{n}(w, z)$ and $v=\sum_{n=0}^{\infty} v_{n}(w, z)$ can be determined as $u(w, z)=\sin w$ and $v(w, z)=\cos w$ So that $u(t, x)=\sin (x+t)$ and $v(t, x)=\cos (x+t)$.

Example 5. Finally, consider Goursat's problem (17)(21) with

$$
\begin{gathered}
\varphi_{0}(x)=\mathrm{e}^{2 x}-1 \text { for } x-t=0 \\
\varphi_{1}(x)=2 \quad \text { for } x-t=0 \\
\varphi_{2}(x)=1-\mathrm{e}^{2 x} \quad \text { for } x+t=0 \\
\varphi_{3}(x)=2 \text { for } x+t=0 \\
f(t, x)=\mathrm{e}^{x+t}-\mathrm{e}^{x-t} \text { and } \lambda=1
\end{gathered}
$$

Then by the above recurrent scheme, we obtain

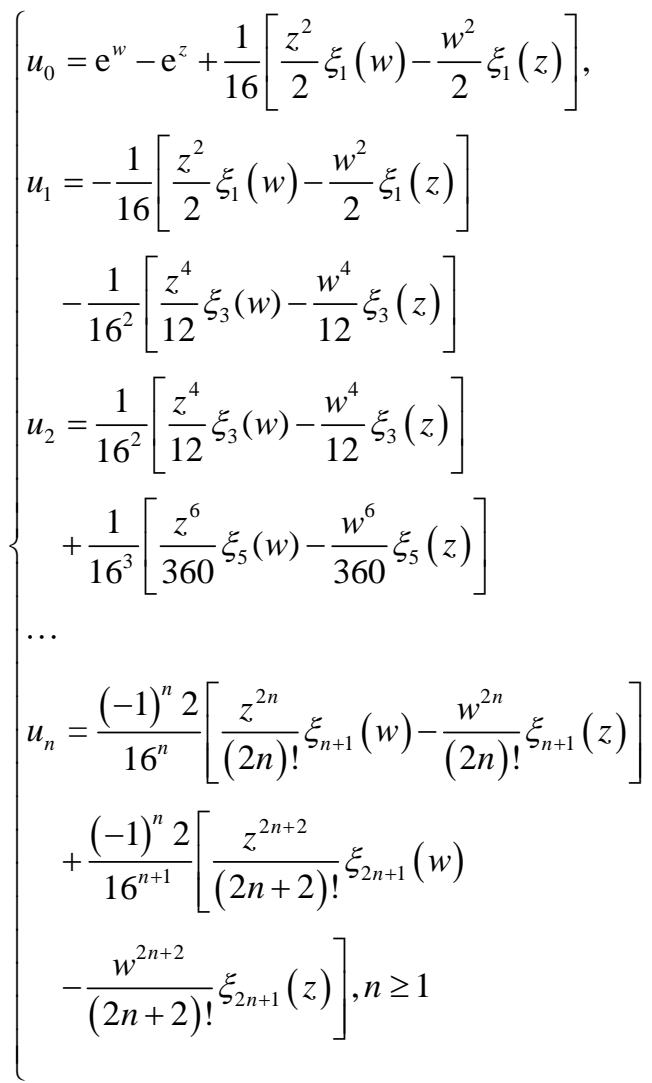

where

$$
\xi_{n}(w)=\mathrm{e}^{w}-\sum_{k=0}^{n} \frac{w^{k}}{k !}
$$

So $u=\sum_{n=0}^{\infty} u_{n}(w, z)$ can be determined as $u(w, z)=\mathrm{e}^{w}-\mathrm{e}^{z}$ Thus $u(t, x)=\mathrm{e}^{x+t}-\mathrm{e}^{x-t}$ which is the exact solution to this problem.

\section{References}

[1] E. Goursat, "A Course in Mathematical Analysis, Vol. 3: Variation of Solutions and Partial Differential Equations of the Second Order and Integral Equations and Calculus of Variations,” Gauthier-Villars, Paris, 1923.

[2] G. Adomian, "Nonlinear Stochastic Operator Equations," Academic Press, Orlando, 1986.

[3] G. Adomian, "Solving Frontier Problems of Physics: The Decomposition Method,” Kluwer Academic Publishers, Boston, 1994.

[4] G. Adomian and R. Rach, "Transformation of Series," Applied Mathematics Letters, Vol. 4, No. 4, 1991, pp. 6971. doi:10.1016/0893-9659(91)90058-4

[5] G. Adomian, R. Rach and R. E. Meyers, “A Modified Decomposition," Computers \& Mathematics with Applications, Vol. 23, No. 1, January 1992, pp. 17-23. doi:10.1016/0898-1221(92)90076-T

[6] G. Adomian and R. Rach, "Inhomogeneous Nonlinear Partial Differential Equations with Variable Coefficients," Applied Mathematics Letters, Vol. 5, No. 2, March 1992, pp. 11-12. doi:10.1016/0893-9659(92)90101-E

[7] G. Adomian and R. Rach, "Nonlinear Transformation of Series Part II," Computers \& Mathematics with Applications, Vol. 23, No. 10, May 1992, pp. 79-83. doi:10.1016/0898-1221(92)90058-P

[8] G. Adomian and R. Rach, "Modified Decomposition Solution of Nonlinear Partial Differential Equations," Applied Mathematics Letters, Vol. 5, No. 6, November 1992, pp. 29-30. doi:10.1016/0893-9659(92)90008-W

[9] G. Adomian and R. Rach, "Solution of Nonlinear Partial Differential Equations in One, Two, Three, and four Dimensions," World Scientific Series in Applicable Analysis, Vol. 2, 1993, pp. 1-13.

[10] G. Adomian and R. Rach, "Modified Decomposition Solution of Linear and Nonlinear Boundary-Value Problems,” Nonlinear Analysis, Vol. 23, No. 5, September 1994, pp. 615-619. doi:10.1016/0362-546X(94)90240-2

[11] G. Adomian and R. Rach, "Analytic Solution of Nonlinear Boundary-Value Problems in Several Dimensions by Decomposition," Journal of Mathematical Analysis and Applications, Vol. 174, No. 1, 15 March 1993, pp. 118137. doi:10.1006/jmaa.1993.1105

[12] G. Adomian and R. Rach, "A New Algorithm for Matching Boundary Conditions in Decomposition Solutions," Applied Mathematics and Computation, Vol. 58, No. 1, September 1993, pp. 61-68. doi:10.1016/0096-3003(93)90012-4

[13] A. Wazwaz, "The Decomposition Method for Approximate Solution of the Goursat Problem," Applied Mathematics and Computation, Vol. 69, No. 2-3, May 1995, pp. 299-311. doi:10.1016/0096-3003(94)00137-S 